University of Nebraska - Lincoln

DigitalCommons@University of Nebraska - Lincoln

February 1969

\title{
Projected Hartree Product Wavefunctions. II. General Considerations of Young Operators
}

Gordon A. Gallup

UNL,ggallup1@unl.edu

Follow this and additional works at: https://digitalcommons.unl.edu/physicsgallup

Part of the Physics Commons

Gallup, Gordon A., "Projected Hartree Product Wavefunctions. II. General Considerations of Young Operators" (1969). Gordon Gallup Publications. 22.

https://digitalcommons.unl.edu/physicsgallup/22

This Article is brought to you for free and open access by the Research Papers in Physics and Astronomy at DigitalCommons@University of Nebraska - Lincoln. It has been accepted for inclusion in Gordon Gallup Publications by an authorized administrator of DigitalCommons@University of Nebraska - Lincoln. 


\title{
Projected Hartree Product Wavefunctions. II. General Considerations of Young Operators*
}

\author{
G. A. Gallup $\dagger$ \\ Quantum Theory Project, University of Florida, Gainesville, Florida 32601
}

(Received 11 March 1968)

\begin{abstract}
A discussion is given of the forms of two specific and one general Young operator for the irreducible representations of $S_{n}$ important for fermion space functions. Comparisons are made of the projected Hartree product version of Löwdon's projected Hartree-Fock method with CI calculations.
\end{abstract}

\section{INTRODUCTION}

Although the connections between the symmetric groups and antisymmetric eigenfunctions of the total spin have been known many years, ${ }^{1-5}$ it is only in recent times that investigations using these methods have been applied to spin-free problems ${ }^{6,7}$ and computation of matrix elements. ${ }^{5}$ In a previous article ${ }^{8}$ hereafter called $I$, the author has given a discussion of the use of Young operators in a spin-free version of the projected Hartree-Fock problem. ${ }^{9}$ Goddard has recently given a discussion of this problem using methods of the symmetric groups, ${ }^{10}$ and Poshusta and Kramling ${ }^{11}$ have given a discussion based on the concept of immanents. ${ }^{2}$

The interest in the independent-particle model arises from the feeling that wavefunctions constructed from single-particle orbitals will be of the simplest type as far as physical interpretations are concerned. In spite of the differences in the details of the computations, all independent-particle model wavefunctions consist of what is essentially a projected Hartree product of spatial orbitals. In I it was shown that use of just one Young operator from the symmetric group $S_{n}$ can guarantee that the function represent a state of pure

* This work was supported by the Research Council of the University of Nebraska and the National Science Foundation through a Science Development Grant to the Chemistry Department, University of Nebraska.

$\dagger$ On leave during academic year 1967-1968 from Department of Chemistry, University of Nebraska, Lincoln, Nebraska 68508.

${ }^{1} \mathrm{H}$. Weyl, Theory of Groups and Quantum Mechanics (Dover Publications, Inc., New York, 1928).

2 E. P. Wigner, Group Theory (Academic Press Inc., New York, 1959)

${ }^{3}$ R. Serber, Phys. Rev. 45, 461 (1934).

${ }^{4}$ T. Yamanouchi, Proc. Phys.-Math. Soc. Japan 20, 547 (1938).

${ }^{5} \mathrm{M}$. Kotani, A. Amemiya, E. Ishiguro, and T. Kimura, Table of Molecular Integrals (Maruzen, Tokyo, 1955).

6 F. A. Matsen in Advances in Quantum Chemistry (Academic Press Inc. New York 1964), Vol. 1; J. Phys. Chem. 68, 3282 (1964) ; 70, 1568 (1966).

${ }_{7}$ F. A. Matsen, A. A. Cantu, and R. D. Poshusta, J. Phys. Chem. 70, 1558 (1966).

${ }^{8}$ G. A. Gallup, J. Chem. Phys. 48, 1752 (1968)

${ }^{9}$ P.-O. Löwdin, Phys. Rev. 97, 1509 (1955), see also, article in Quantum Theory of Atoms Molecules and the Solid State, P.-O. Löwdin, Ed. (Academic Press Inc., New York, 1966), p. 601.

${ }^{10}$ W. A. Goddard, Phys. Rev. 157, 73, 81, 93 (1967), J. Chem. Phys. 48, 450, 1008 (1968).

${ }_{11}$ R. D. Poshusta and R. W. Kramling, Phys. Rev. 167, 139 (1968).

${ }_{12}$ D. E. Littlewood, Theory of Group Characters (Oxford University Press, London, 1950), 2nd ed. multiplicity for a system of $n$ electrons, and these functions can be used to compute matrix elements of spin-free operators. A self-consistant field computation based on these Young operators was also discussed with some applications to simple systems.

In this article we give some detailed properties of the Young operators needed for calculations of the type given in $\mathrm{I}$ and indeed for any computations of matrix elements of spin-free operators with respect to antisymmetric eigenfunctions of the spin. We also discuss the relationship among some of the methods for writing antisymmetric eigenfunctions of the spin, some comparisons of projected Hartree product wavefunctions and CI computations, and the some effects of assumed orthogonality between various spatial orbitals.

\section{SPECIFIC YOUNG OPERATORS}

In this section we shall examine two specific forms for a Young operator corresponding to the irreducible representations of the symmetric group for eigenfunctions of the total spin angular momentum. We are interested, of course, in the idempotents appropriate for the spatial part of the wavefunction but we may analyze either the two-row tableaux (partitions) or the two-column tableaux, since these cases are merely conjugate. We shall choose as the basis for this article the tableaux corresponding to $\left(2^{(n / 2)-S}, 1^{2 S}\right)$, for spatial functions appropriate to the spin quantum number $S$.

Littlewood ${ }^{12}$ shows that the idempotents $P$ and $N$ each have an intersection of rank 1 with the $\left(2^{n / 2-s}, 1^{2 S}\right)$ subalgebra and no other parts in common. Hence $P N P$ and $N P N$ are each proportional to a Hermitian primitive characteristic unit of $\left(2^{(n / 2)-S}, 1^{2 S}\right)$ as was pointed out in $I$. It was also shown in $I$ that a primitive characteristic unit can be factored, and we wish to calculate the factored form of $\theta N P N$ and $\theta^{\prime} P N P$,

$$
e^{(\mu)}=\frac{f(\mu)}{|G|} E \sum_{i=1}^{k} a\left(X_{i}\right) X_{i} .
$$

We will call the subgroup with elements in $E$ the base subgroup and denote it by $G_{B}$. From Eq. (1) we obtain immediately

$$
\sum_{i=1}^{k}\left|a\left(X_{i}\right)\right|^{2}=\frac{|G|}{\left|G_{B}\right| f_{(\mu)}} .
$$


We may also make the general statement that the necessary and sufficient condition that an element of $G$ be in $G_{B}$ is that it commutes with $e^{(\mu)}$. We take up the computation of $\theta N P N$ first since it is a little simpler.

The factorization of a characteristic unit as in Eq. (1) is very important, since it provides easy access of some other results as we shall see later.

The coefficients of the permutations in the operators $N P N$ and $P N P$ are very important for the computation of matrix elements and also for the determination of the general idempotent discussed in Sec. IV. Matsen ${ }^{6}$ has also discussed the operators $N P N$ and $P N P$ from a somewhat different point of view.

\section{A. The Operator $\theta$ NPN}

For purposes of discussion we assume we have a tableau for $\left(2^{n / 2-S}, 1^{2 S}\right)$ with numbers inserted as shown in Fig. ${ }^{13}$ It is evident that all elements of $N$ commute with $N P N$ and are part of $G_{B}$. If $S \neq 0$ then no other element of $S_{n}$ commutes with $N P N$ and $G_{N}=G_{B}$. When $S=0, n$ is an even integer, and one element from $P$ will commute with $N P N$. This element is $(1, n / 2+1)(2,(n / 2+2) \cdots(n / 2, n)$ which we may denote $p$ and $G_{B}=\left\{G_{N}, p G_{N}\right\}$. Thus we obtain

$$
\begin{aligned}
\left|G_{B}\right| & =[(n / 2)+S] ![(n / 2)-S] ! ; & & S \neq 0, \\
& =2[(n / 2) !]^{2} ; & & S=0,
\end{aligned}
$$

and Eq. (2) becomes

where

$$
\begin{aligned}
\sum_{i=1}^{k}\left|a\left(X_{i}\right)\right|^{2} & =\frac{n+2 S+2}{4 S+2} ; & & S \neq 0 \\
& =(n+2) / 4 ; & S & =0,
\end{aligned}
$$

$$
k=\left(\begin{array}{c}
n \\
(n / 2)+S
\end{array}\right)
$$

or

$$
\frac{1}{2}\left(\begin{array}{c}
n \\
n / 2
\end{array}\right)
$$

If $N$ and $P$ are each normalized so that the coefficient of $I$ in each is 1 , then

$$
\theta=f_{(\mu)} /\left|G \| G_{N}\right|
$$

and $a\left(X_{i}\right)$ is the number of times $X_{i}$ appears in the
FIG. 1. Numbering for the general tableau $T$.

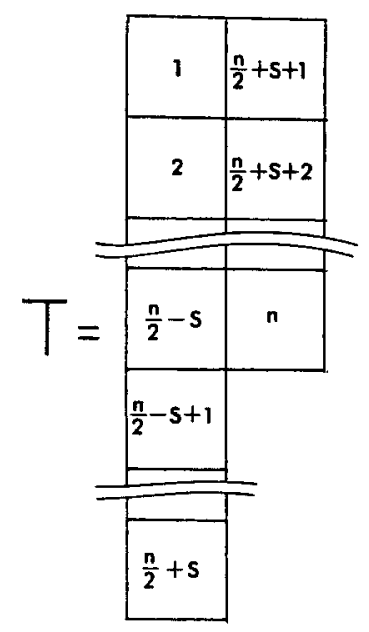

product $N P N$ divided by $\left|G_{N}\right|$ with the appropriate sign affixed.

Consider an element of the class $\left(1^{n-2 l}, 2^{l}\right)$ of $S_{n}$ with $l \leq(n / 2)-S$. Such a permutation may have each interchange $(e, f)$ such that $e$ is from the first column and $f$ is from the second column of the tableau $T$. We shall see that a complete set of right coset generators may be obtained from the type of permutation under consideration. For definiteness let $i_{1}, i_{2}, i_{3}, \cdots, i_{l}=(i)$ be a set of $l$ different entries from the first column of $T$, such that $i_{1}<i_{2}<\cdots<i_{l}$. Let $j_{1}, j_{2}, \cdots, j_{l}=(j)$ be a set from the second column similarly ordered. These $2 l$ numbers determine a permutation from the class $\left(1^{n-2 l}, 2^{l}\right)$,

$$
[(i)(j)]=\left(i_{1} j_{1}\right)\left(i_{2} j_{2}\right) \cdots\left(i_{l} j_{l}\right) .
$$

It is easily seen that $[(i)(j)]$ appears

$$
l ![(n / 2)+S-l] ![(n / 2)-S] !
$$

times in $N P N$, hence

$$
a([(i)(j)])=\left(\begin{array}{c}
(n / 2)+S \\
l
\end{array}\right)^{-1}
$$

This result is true regardless of the value of $S$. There are in all $l$ ! permutations of $\left(1^{n-2 l}, 2^{l}\right)$ connecting the set $(i)$ with the set $(j)$. However, it may be shown that these all are in the same coset, hence the specific permutation defined by Eq. (4) is the only one needed. We may put these results together and obtain

$$
\begin{aligned}
e^{(\mu)} & =\frac{2 S+1}{(n+1) !}\left(\begin{array}{c}
n+1 \\
(n / 2)-S
\end{array}\right) N\left\{I+\sum_{l=1}^{(n / 2)-S} \sum_{(i)} \sum_{(j)}\left(\begin{array}{c}
(n / 2)+S \\
l
\end{array}\right)^{-1}[(i)(j)]\right\} ; \quad S \neq 0, \\
& =[(n+1) !]^{-1}\left(\begin{array}{c}
n+1 \\
n / 2
\end{array}\right) N\{I+p\}\left\{I+\sum_{l=1}^{k} \sum_{(i)} \sum_{(j)}\left(\begin{array}{c}
(n / 2) \\
l
\end{array}\right)^{-1}[(i)(j)]\right\} ; \quad S=0,
\end{aligned}
$$

${ }^{13}$ D. E. Rutherford, Substitutional Analysis (Edinburgh University Press, London, 1948). 


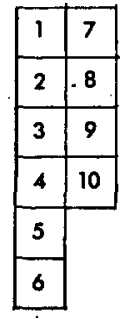

FIG. 2. Tableau for the triplet case of ten electrons.

where $k$ is $n / 4$ or $(n-2) / 4$, whichever is integral. It is not difficult to see that we have found all the coset generators and that Eq. (2) is satisfied.

As we shall see later $\theta N P N$ is a rather special form for $e^{(\mu)}$.

\section{B. The Operator $\theta^{\prime}$ PNP}

In this case it is seen that $G_{B}$ contains all elements of $P$, but it also contains all elements of $N$ which commute with $P$. Considering the same tableau $T$ as before, all permutations of the numbers $(n / 2)-S+1,(n / 2)-$ $S+2, \cdots,(n / 2)+S$ do not involve any numbers in the elements of $P$, hence these are part of $G_{B}$. There are $(2 S)$ ! such permutations. The other permutations of $N$ which are in $G_{B}$ are products of the same permutation of $1,2, \cdots,(n / 2)-S$ and $(n / 2)+S+1,(n / 2)+$ $S+2, \cdots, n$. Thus in this case we have

$$
\left|G_{B}\right|=2^{(n / 2)-S}[(n / 2)-S] !(2 S) ! .
$$

The coset generators are somewhat more difficult to characterize in this case although we still need only elements from the class $\left(1^{n-2 l}, 2^{l}\right)$ for the singlet case. To make the description simpler we define a few terms: Let us call the first $(n / 2)-S$ rows of $T$ the body and the other part the tail. We shall call an element of $\left(1^{n-2 l}, 2^{l}\right)$ a simple chain if it may be written $\left(i_{1} j_{1}\right)\left(i_{2} j_{2}\right) \cdots\left(i_{l} j_{l}\right)$ and each of the pairs of numbers $j_{1}, i_{2} ; j_{2}, i_{3} ; \cdots ; j_{L_{1}} i_{l}$ is taken from the same row of the body. For example in the tableau in Fig. 2, $(72)(89)(35)$ and $(91)(72)(84)(10,6)$ are simple chains. If $i_{1}$ and $j_{l}$ are also in the same row we have a loop. It is evident that a loop can contain no entries

TABLE I. Right coset generators and coefficients for two-column partitions of $S_{4}{ }^{\mathrm{s}}$

\begin{tabular}{ccccc}
\hline \hline \multicolumn{2}{c}{$\left(21^{2}\right)$} & & & \multicolumn{2}{c}{$\left(2^{2}\right)$} \\
\cline { 1 - 2 } \cline { 5 - 5 } & $a(X)$ & & $X$ & $a(X)$ \\
\hline$I$ & 1 & & $I$ & 1 \\
$(12)$ & $-\frac{1}{2}$ & & $(12)$ & $-\frac{1}{2}$ \\
$(13)$ & $-\frac{1}{2}$ & & $(14)$ & $-\frac{1}{2}$ \\
$(24)$ & $-\frac{1}{2}$ & & & \\
$(34)$ & $-\frac{1}{2}$ & & & \\
$(12)(34)$ & 0 & & \\
\hline
\end{tabular}

- These results are based on the numbering of Fig. 1. from the tail and a simple chain can contain no more than two entries from the tail. A composite chain is made up of two or more disconnected simple chains. We shall consider only the singlet case $(S=0)$ here.

We use the normalization of $P$ and $N$ as before and again $a\left(X_{i}\right)$ is the number of times $X_{i}$ appears in $P N P$ divided by $2^{(n / 2)-S}$ with the appropriate sign. It is not too difficult to see that for $S=0$, any composite or simple chain in the class $\left(1^{n-2 l}, 2^{l}\right)$ has a coefficient $(-2)^{-l}$. If a composite chain $K_{l}$ is made up of simple chains of lengths $l_{1}, \cdots, l_{i}$, we denote it $K_{l}\left(l_{1} l_{2} \cdots l_{i}\right) ; l_{1}+l_{2}+\cdots+l_{i}=l$. In each coset there

TABLE II. Right coset generators and coefficients for two-column partitions of $S_{6}$."

\begin{tabular}{ccccc}
\hline \hline & $\left(21^{3}\right)$ & & $\left(2^{2} 1\right)$ & \\
\cline { 1 - 2 } \cline { 5 - 5 }$X$ & $a(X)$ & & $X$ & $a(X)$ \\
\hline$I$ & 1 & & 1 \\
$(12)$ & $-\frac{1}{2}$ & & $(12)$ & $-\frac{1}{2}$ \\
$(13)$ & $-\frac{1}{2}$ & & $(15)$ & $-\frac{1}{2}$ \\
$(14)$ & $-\frac{1}{2}$ & & $(13)$ & $-\frac{1}{2}$ \\
$(25)$ & $-\frac{1}{2}$ & & $(23)$ & $-\frac{1}{2}$ \\
$(35)$ & $-\frac{1}{2}$ & & $(34)$ & $-\frac{1}{2}$ \\
$(45)$ & $-\frac{1}{2}$ & & $(35)$ & $-\frac{1}{2}$ \\
$(12)(35)$ & 0 & & $(12)(34)$ & $\frac{1}{4}$ \\
$(12)(45)$ & 0 & & $(12)(35)$ & $\frac{1}{4}$ \\
$(13)(45)$ & 0 & & $(13)(45)$ & $\frac{1}{4}$ \\
& & & $(23)(45)$ & $\frac{1}{4}$ \\
& & & $(15)(23)$ & $\frac{1}{6}$ \\
& & $(15)(34)$ & $\frac{1}{6}$ \\
& & $(13)(24)$ & $\frac{1}{4}$ \\
& & $(24)(35)$ & $\frac{1}{4}$ \\
\hline \hline
\end{tabular}

These results are based on the numbering of Fig. 1.

are $\left(l_{1}+1\right)\left(l_{2}+1\right) \cdots\left(l_{i}+1\right)$ such chains. We obtain for $S=0$

$$
\begin{aligned}
e^{(\mu)}= & {[(n+1) !]^{-1}\left(\begin{array}{c}
n+1 \\
n / 2
\end{array}\right) G_{B} } \\
& \times\left\{I+\sum_{l=1}^{(n / 2)-1}\left[(-1)^{l} / 2^{l}\right] \sum_{l_{1} \cdots l_{i}}^{\prime} K_{l}\left(l_{1} \cdots l_{i}\right)\right\},
\end{aligned}
$$

where the $\sum$ 'indicates the sum is over only the chains generating distinct cosets. Tables I-III give the coefficients and right coset generators of $\theta^{\prime} P N P$ for two column partitions of $S_{4}, S_{5}$, and $S_{6}$.

\section{THE PROJECTED SLATER DETERMINANT}

The primitive characteristic unit in the form $\theta N P N$ has a rather special form which is connected with the 
TABLE III. Right coset generators and coefficients for two-column partitions of $S_{6 .}{ }^{*}$

\begin{tabular}{|c|c|c|c|c|c|}
\hline \multicolumn{2}{|c|}{$\left(21^{4}\right)$} & \multicolumn{2}{|c|}{$\left(2^{2} 1^{2}\right)$} & \multicolumn{2}{|c|}{$\left(2^{3}\right)$} \\
\hline$X$ & $a(X)$ & $X$ & $a(X)$ & $X$ & $a(X)$ \\
\hline $\begin{array}{c}I \\
(12) \\
(13) \\
(14) \\
(15) \\
(26) \\
(36) \\
(46) \\
(56) \\
(12)(36) \\
(12)(46) \\
(12)(56) \\
(13)(46) \\
(13)(56) \\
(14)(56)\end{array}$ & $\begin{array}{r}1 \\
-\frac{1}{2} \\
-\frac{1}{2} \\
-\frac{1}{2} \\
-\frac{1}{2} \\
-\frac{1}{2} \\
-\frac{1}{2} \\
-\frac{1}{2} \\
-\frac{1}{2} \\
0 \\
0 \\
0 \\
0 \\
0 \\
0\end{array}$ & $\begin{array}{c}I \\
(13) \\
(14) \\
(23) \\
(24) \\
(35) \\
(45) \\
(36) \\
(46) \\
(12) \\
(16) \\
(12)(35) \\
(12)(45) \\
(12)(36) \\
(12)(46) \\
(16)(23) \\
(16)(24) \\
(16)(35) \\
(16)(45) \\
(25)(13) \\
(25)(14) \\
(25)(36) \\
(25)(46) \\
(56)(13) \\
(56)(14) \\
(56)(23) \\
(56)(24) \\
(13)(46) \\
(14)(36) \\
(23)(45) \\
(24)(35) \\
(13)(24) \\
(14)(23) \\
(35)(46) \\
(36)(45) \\
(13)(45) \\
(13)(245) \\
(13)(456) \\
(123)(45) \\
(163)(45) \\
(23)(46) \\
(23)(146) \\
(23)(465) \\
(132)(46) \\
(253)(46)\end{array}$ & 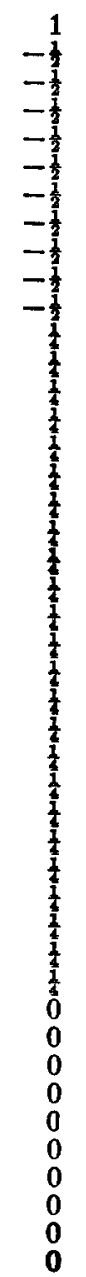 & $\begin{array}{c}I \\
(12) \\
(13) \\
(23) \\
(15) \\
(26) \\
(34) \\
(12)(46) \\
(12)(34) \\
(15)(46) \\
(16)(24) \\
(13)(45) \\
(16)(45) \\
(13)(24) \\
(15)(34)\end{array}$ & $\begin{array}{r}1 \\
-\frac{1}{2} \\
-\frac{2}{2} \\
-\frac{1}{2} \\
-\frac{1}{2} \\
-\frac{1}{2} \\
-\frac{1}{2} \\
\frac{1}{2} \\
\frac{1}{\frac{1}{2}} \\
\frac{1}{\frac{1}{2}} \\
\frac{1}{2} \\
\frac{1}{2} \\
\frac{1}{4}\end{array}$ \\
\hline
\end{tabular}

These results are based on the numbering of Fig. 1.

irreducible representations of $S_{n}$ obtained from the spin basis functions produced by any spin projection operators which commutes with all permutations. Let us assume that we have a product spin function

$\psi(n, S)=\alpha(1) \alpha(2) \cdots \alpha[(n / 2)+S]$

$$
\times \beta[(n / 2)+S+1] \cdots \beta(n) .
$$

If we have a projection operator $\theta_{S}$ which satisfies $\left[\theta_{S}, \pi\right]=0$ for every permutation of $S_{n}$ we may construct a spin function for the principle case $M=S^{14}$

$$
\varphi_{1}(S, S)=C \theta_{s} \psi(n, S) .
$$

We label the function defined by Eq. (8) 1. It is well

${ }^{14}$ P.-O. Löwdin, Rev. Mod. Phys. 36, 966 (1964). known that

$$
\frac{2 S+1}{n+1}\left(\begin{array}{c}
n+1 \\
(n / 2)-S
\end{array}\right)-1
$$

other spin functions may be constructed orthogonal to $\varphi_{1}(S, S)$ and to each other, $\varphi_{2}(S, S), \cdots, \varphi_{f}(S, S)$. We assume that the functions are all normalized. The $f$ functions form a basis for an irreducible representation of $S_{n}$;

$$
\pi \varphi_{i}(S, S)=\sum_{j} D^{(S)}(\pi)_{j i \varphi_{j}}(S, S),
$$

and we want to obtain

$$
e_{11}^{(S)}=(f / g) \sum_{x} D^{(S)}(\pi)_{11} \pi,
$$


where

$$
D^{(S)}(\pi)_{11}=\left\langle\varphi_{1}(S, S)|\pi| \varphi_{1}(S, S)\right\rangle .
$$

The product function $\psi(n, S)$ determines a two-row tableau which may be taken as the conjugate of $T, \bar{T}$. We see that the elements of $P$ of $\bar{T}$ are such that $\pi_{P} \psi(n, S)=\psi(n, S)$ and hence $D^{(S)}\left(\pi_{P}\right)_{11}=1$, and $G_{B}$ for $e_{11}^{(S)}$ is at least $P$ of $\bar{T}$. But this is sufficient to prove that $e_{11}{ }^{(S)}=\theta P N P$ for $\tilde{T}$. Taking the conjugate we obtain exactly

$$
e^{(\mu)}=e_{11}{ }^{(S)}=\theta N P N
$$

or Eq. (1). For this reason use of the projector $\theta N P N$ for $T$ is equivalent to using a spin projection operator on a single Slater determinant with $M=S$. McIntosh ${ }^{15}$ has obtained an essentially equivalent result by a different method. The operator $\theta^{\prime} P N P$ gives no such simple result, of course. It may be observed also that $\theta^{\prime} P N P$ and $\theta N P N$ correspond to Goddard's $G 1$ and $G F$ methods, respectively. ${ }^{10}$

The operator $\theta N P N$ is interesting in another light, since the result of its application to a product of spatial functions is closely connected with a form of determinantal wavefunction originated by $\mathrm{Hartree}^{16}$ and used by Wigner. ${ }^{17}$ The function

$$
\Psi=N u_{1}(1) u_{2}(2) \cdots u_{n}(n)
$$

is just Hartree's double determinantal function and it is interesting that the further application of $P$ gives a function appropriate for a given spin.

\section{A GENERAL CHARACTERISTIC UNIT}

In I it was mentioned that the most general function of the correct symmetry that can be obtained from a single product function is

$$
\Psi=e^{(\mu)} \sum_{i} \gamma_{i} X_{i}|(\lambda)\rangle,
$$

where $X_{i}$ are certain selected permutations from the right coset generators of the $G_{B}$ for $e^{(\mu)}$. There are a number of ways the $X_{i}$ in Eq. (10) may be chosen but one of the simplest is by considering the various standard tableaux [with or without repeated elements, depending on $(\lambda)]$ and the permutation changing $T$ into the others. We may assume without loss of generality that if $(\lambda) \neq(\mu)$ the repeated functions are in the first rows of $T$, then we have $k=t_{(\mu)}{ }^{(\lambda)}$ standard tableaux $T_{1}(=T), T_{2}, \cdots, T_{k}$ and the permutations $\sigma_{i j}$ such that

$$
T_{1} \sigma_{1 j}=\sigma_{1 j} T_{j} .
$$

If the tableaux are ordered in the standard fashion (12), the relation

$$
\theta N P N \sum_{j=1}^{k} \gamma_{j} \sigma_{1 j}=0
$$

${ }^{15}$ H. V. McIntosh, J. Math. Phys. 1, 453 (1960).

${ }^{16}$ D. R. Hartree, Proc. Cambridge Phil. Soc. 24, 89 (1928).

17 E. P. Wigner, Phys. Rev. 46, 1002 (1934). implies $\gamma_{j}=0$. This result is shown in Appendix C. Similar considerations apply to $\theta^{\prime} P N P$. We may construct an equivalence relation between the $\sigma_{1 j}$ and certain of the right coset generators, since $\sigma_{1 j}$ is in some coset of $G_{B}$ and

$$
G_{B} \sigma_{1 j}=\epsilon G_{B} X_{k} ; \quad|e|=1,
$$

and it is evident that the $X_{k}$ may be used in Eq. (11) in place of the $\sigma_{1 j}$.

The primitive characteristic unit $e^{(\mu)}$ has a rank 1 . This implies that any element of the algebra of the form

$$
y=e^{(\mu)} \sum_{j=1}^{f(\mu)} \gamma_{j} X_{j},
$$

is essentially idempotent, i.e.,

$$
y^{2}=\lambda y,
$$

in which $y \neq y^{\dagger}$, in general. We may construct a form for the most general Hermitian, primitive, characteristic unit as $y^{\dagger} y / S(\gamma)$, where $f_{(\mu)} S(\gamma) /|G|$ is the coefficient of the identity in

$$
\sum_{j=1}^{f(\mu)} \sum_{k=1}^{f(\mu)} \gamma_{j}{ }^{*} \gamma_{k} X_{j}^{\dagger} e^{(\mu)} X_{k}
$$

$S(\gamma)$ can be written in matrix notation as

$$
S(\boldsymbol{\gamma})=\boldsymbol{\gamma}^{\dagger} \boldsymbol{S}_{\boldsymbol{\gamma}},
$$

and it is easily shown that $\mathbf{S}$ is a positive definite matrix since the $e^{(\mu)} X_{j}$ are linearly independent. We shall denote $y^{\dagger} y / S(\gamma)$ as $E^{(\mu)}(\gamma)$, and examine more closely its form. In general,

$$
E^{(\mu)}(\gamma)=\left(f_{(\mu)} /|G|\right) \sum_{\boldsymbol{\tau}} P(\gamma, \pi) \pi,
$$

where $P(\gamma, \pi)$ is a rational expression involving polynomials in $\gamma$ in the numerator and $S(\gamma)$ in the denominator. If we return to the our original idempotent $e^{(\mu)}$,

$$
e^{(\mu)}=\left(f_{(\mu)} /|G|\right) \sum_{\pi} a(\pi) \pi,
$$

TABLE IV. $P(\gamma, \pi)$ coefficients for general idempotent for $(2,1)$ of $S_{3}$.

\begin{tabular}{ll}
\hline \hline$\pi$ & \multicolumn{1}{c}{$P(\gamma, \pi)$} \\
\hline$I$ & 1 \\
$(12)$ & $\left(-\gamma_{1}^{3}-\gamma_{1} \gamma_{2}+\frac{1}{2} \gamma_{2}{ }^{2}\right) / S(\gamma)$ \\
$(13)$ & $\left(\frac{1}{2} \gamma^{2}+2 \gamma_{1} \gamma_{2}+\frac{1}{2} \gamma_{2}^{2}\right) / S(\gamma)$ \\
$(23)$ & $\left(\frac{1}{2} \gamma_{1}^{2}-\gamma_{1} \gamma_{2}-\gamma_{2}{ }^{2}\right) / S(\gamma)$ \\
$(123)$ & $-\frac{1}{2}$ \\
$(132)$ & $-\frac{1}{2}$ \\
& $S(\gamma)=\gamma_{1}^{2}+\gamma_{1} \gamma_{2}+\gamma_{2}{ }^{2}$ \\
\hline
\end{tabular}


we may write $P(\gamma, \pi)$ in terms of the $a$ 's as

$$
\begin{aligned}
& P(\gamma, \pi) \\
& =\left[\sum_{j, k} \gamma_{j}{ }^{*} \gamma_{k} a\left(X_{k}{ }^{-1} \pi^{-1} X_{j}\right)\right] / \sum_{j, k} \gamma_{j}{ }^{*} \gamma_{k} a\left(X_{k}{ }^{-1} X_{j}\right) .
\end{aligned}
$$

Table IV give an example of $E^{(\mu)}(\gamma)$ for the $(2,1)$ representation of $S_{3}$. Appendix A gives a method for evaluating $a(\pi)$ for $\theta N P N$. Equation (13) is more general than our purposes require, since we have chosen our coset generators from the class $\left(1^{n-2 l}, 2^{l}\right)$. Thus, these elements are involuntary, and the inverses are unnecessary.

It is evident from the foregoing that the four common ways in which the spin-degeneracy problem may be handled are completely equivalent. For purposes of illustration let us assume we have a set of $n$ oneelectron, spatial orbitals. As was shown by Löwdin ${ }^{14}$ the Slater determinantal functions constructed from these orbitals and the spin functions and corresponding to points in the upper half of a "path diagram" yield linearly independent functions after being subjected to a spin projection operator. Thus, if the determinants are denoted $D_{1}, D_{2}, \cdots, D_{k}$,

$$
\Theta_{S} \sum_{i=1}^{k} \gamma_{i}^{\prime \prime} D_{i}=\Psi_{S}
$$

is most general spin eigenfunction that can be formed from the spatial orbitals given.

Larsson $^{18}$ has used another procedure. Let $\theta_{1}$, $\theta_{2}, \cdots, \theta_{k}$ be the $k$ pure spin eigenfunctions for $n$ fermions and spin $S$, and $\Phi(1,2, \cdots, n)$ be the product of our $n$ spatial orbitals. The function

$$
\Psi_{S}=\mathscr{A} \Phi(1,2, \cdots, n) \sum_{i=1}^{k} \gamma_{i}^{\prime} \theta_{i},
$$

is the same as that given by Eq. (14), where $\mathcal{A}$ is the antisymmetrizer. The numbers $\gamma_{i}^{\prime \prime}$ and $\gamma_{i}^{\prime}$ are related by a linear transformation, of course.

Paunc ${ }^{19}$ and Harris ${ }^{20}$ have pointed out that sums of various permutations of the $\Phi$ in Eq. (15) multiplied by just one $\theta$ will when antisymmetrized give $\Psi_{S}$, viz.,

$$
\Psi_{S}=\mathcal{A} \Theta \sum_{i=1}^{k} \gamma_{i}^{\prime \prime \prime} \pi_{i} \Phi(1,2, \cdots, n),
$$

and the $\gamma_{i}^{\prime \prime \prime}$ are linearly related to $\gamma_{i}^{\prime \prime}$ and $\gamma_{i}^{\prime}$.

The fourth method here under discussion for spinfree operator yields

$$
\Psi^{(\mu)}=E^{(\mu)}(\gamma) \Phi(1,2, \cdots, n),
$$

which for a set $\gamma_{i}$ linearly related to $\gamma_{i}{ }^{\prime}, \gamma_{i}{ }^{\prime \prime}$, or $\gamma_{i}{ }^{\prime \prime}$ will give an energy equal to that calculated from $\Psi_{S}$ in Eqs. (14), (15), or (16) for $(\mu)=\left(2^{(n / 2)-S}, 1^{2 S}\right)$.

${ }^{18} \mathrm{~S}$. Larsson, "Calculations on the ${ }^{2} S$ Ground State of the Lithium Atom Using Wavefunctions of the Hylleraas Type," Uppsala Quantum Chemistry Group Tech. Note No. 197 (1967).

${ }^{19}$ R. Pauncz, J. Chem. Phys. 43, S69 (1965)

${ }^{20}$ F. E. Harris, Advan. Quantum Chem. 3, 61 (1966).
The forms given in Eqs. (15) $-(17)$ are more easily generalized to nonproduct functions for $\Phi$, of course. We may say that for a given $\Phi(1,2, \cdots, n)$ there is a best spin function $\sum_{i} \gamma_{i}^{\prime} \theta_{i}$ for form (15) or a best $E^{(\mu)}(\gamma)$ for form (17) to give the lowest calculated value of the ground-state energy. As an example it is seen from I that the $E^{(2,1)}(\gamma)$ giving the "full" configuration function for the allyl radical is

$$
\begin{array}{r}
E^{(2,1)}(\gamma)=\frac{1}{3}\{I+0.901(12)- \\
-0.074(13)-0.826(23) \\
\left.-\frac{1}{2}(123)-\frac{1}{2}(132)\right\} .
\end{array}
$$

\section{COMPARISON TO CI CALCULATIONS}

Under certain circumstances it is advantageous to look upon projected Hartree product (PHP) methods as restricted CI calculations, when a fixed basis is used for expanding the orbitals. The question arises: When, if ever, is the projected SCF wavefunction equivalent to a full CI wavefunction? As was seen in I, there is equivalence for the $\pi$ system of the allyl radical when the calculation is based on three $p$ orbitals. We can make considerable progress toward answering this question by noting that a sufficient condition for nonequivalence is that the effective number of adjustable parameters in the PHP scheme be less than the number of independent functions constructable from the fixed basis. For a calculation involving $n$ electrons, $m$ basis functions in the fixed basis, a spin of $S$, and no spatial symmetry the number of independent parameters $N_{p}$ in $E^{(\mu)}(\gamma) u_{1}(1) u_{2}(2) \cdots u_{n}(n)$ is

$$
N_{p}=n(m-1)+\frac{2 S+1}{n+1}\left(\begin{array}{c}
n+1 \\
(n / 2)-S
\end{array}\right) \text {. }
$$

The second term on the right of (19) is just the dimension of the vector $\gamma$. The number of independent functions $N_{F}$ is given in Appendix B as

$$
N_{F}=\frac{2 S+1}{m+1}\left(\begin{array}{c}
m+1 \\
(n / 2)+S+1
\end{array}\right)\left(\begin{array}{c}
m+1 \\
(n / 2)-S
\end{array}\right)
$$

The smallest number of functions is had when $m=n$ and

$$
\begin{aligned}
& N_{p}-N_{F}=n(n-1)+\frac{2 S+1}{n+1}\left(\begin{array}{c}
n+1 \\
(n / 2)-S
\end{array}\right) \\
& \times\left[1-\left(\begin{array}{c}
n+1 \\
(n / 2)+S+1
\end{array}\right)\right], \\
& =n(n-1) ; \quad S=n / 2, \\
& =0 ; \quad S=(n / 2)-1 \text {, } \\
& <0 ; \quad S<(n / 2)-1 .
\end{aligned}
$$

Thus there can never be equivalence if $m=n$ and the system is not in one of the two highest multiplicities. Therefore, the result for allyl is somewhat exceptional, since the lowest multiplicity is the next from the highest in any three-electron system. 
In considering PHP methods using a fixed basis for the orbitals it is possible to view the trial function as a sum of configurations with coefficients which are restricted by various relations among them..$^{21}$ As can be seen from Eq. (20) the number of independent eigenfunctions of the spin increases rapidly with $n$ and $m$. It is necessary to choose configurations in the CI method in some manner, and the real test of the PHP methods will be their comparison with such CI computations and with multiconfigurational SCF methods. ${ }^{22,23}$ Such a computation is underway on $\mathrm{H}_{2}$.

\section{ORTHOGONALITY PATTERNS}

For $n \geq 5$ the number of terms in $E^{(\mu)}(\gamma)$ is very large, and it seems necessary with present day computing equipment to make some assumptions which will have a simplifying effect on the energy expression. One way of doing this is to require that certain of the spatial orbitals are orthogonal to one another. There seems to be no $a$ priori method based on energy values for deciding which pattern is best since any such requirement is a restriction which except for one case will in general raise the calculated energy. Therefore, the desideratum is simplication of the energy expression. Let us assume our $n$ spatial orbitals are divided into $p$ subsets the $i$ th containing $l_{i}$ orbitals. One has the equation

$$
\sum_{i} l_{i}=n \text {. }
$$

We denote the $k$ th member of the $i$ th subset $u_{i k}$; $i=1,2, \cdots, p ; k=1,2, \cdots, l_{i}$ and it is assumed that

$$
\left\langle u_{i k} \mid u_{i^{\prime} k^{\prime}}\right\rangle=\delta_{i i}, \Delta(i)_{k k^{\prime}}
$$

that is, each function is orthogonal to every function not in the same subset.

Functions with the property (22) can be used to determine a certain set of conjugate subgroups of $S_{n}$ and we will procede to define one of these. Let us arrange the $u_{i k}$ in dictionary order

$$
\begin{aligned}
u_{11}, u_{12}, \cdots, u_{1 l_{1}} ; u_{21}, u_{22}, \cdots, & u_{2 l_{2}} ; \\
& \times \cdots ; u_{p 1}, u_{p 2}, \cdots, u_{p l_{p}},
\end{aligned}
$$

and let the $j$ th one be a function of spatial coordinates $j$ and form a single-product function by multiplying all of these together,

$$
\phi_{d}(1,2, \cdots, n)=u_{11}(1) u_{12}(2) \cdots u_{p l_{p}}(n) .
$$

An element of $S_{n}$ denoted $\pi$ may operate on $\phi_{d}$ and we may compute the inner product $\left\langle\phi_{d} \mid \pi \phi_{d}\right\rangle=\langle\pi\rangle_{d}$. If none of the $\Delta(i)_{k k}$, are zero then the set of $\pi$ such that $\langle\pi\rangle_{d} \neq 0$ form a subgroup of $S_{n}$, in fact, just $S_{l_{1}} \times$ $S_{l_{2}} \times \cdots \times S_{l_{p}}$, or at least one conjugate to it.

In the following we shall use $\pi_{S}$ to denote an element of $S_{l_{1}} \times \cdots \times S_{l_{p}}$ and $\pi, \sigma, \nu$ etc., for any elements of $S_{n}$.

We wish to evaluate the energy expression for a Hartree product function as given by (23), that is

$$
\mathcal{E}=\left\langle\phi_{d}\left|H E^{(\mu)}(\gamma)\right| \phi_{d}\right\rangle /\left\langle\phi_{d}\left|E^{(\mu)}(\gamma)\right| \phi_{d}\right\rangle .
$$

We shall look at the normalization integral first. Using Eqs. (12) and (22) it is seen that

$$
\left\langle\phi_{d} \mid E^{(\mu)}(\gamma) \phi_{d}\right\rangle=\left(f_{(\mu)} /|G|\right) \sum_{\pi S} P\left(\gamma, \pi_{S}\right)\left\langle\phi_{d}\left|\pi_{S}\right| \phi_{d}\right\rangle
$$

with only $l_{1} ! l_{2} ! \cdots l_{p} !$ terms. The spin-free Hamiltonian $H$ will be assumed to have only one- and two-particle operators,

$$
\begin{aligned}
H & =F+G \\
& =\sum_{m} f_{m}+\sum_{m<n} g_{m n},
\end{aligned}
$$

and we have immediately that

$$
\begin{aligned}
& \left\langle\phi_{d}\left|F E^{(\mu)}(\gamma)\right| \phi_{d}\right\rangle \\
& \quad=\left(f_{(\mu)} /|G|\right) \sum_{\pi_{S}} P\left(\gamma, \pi_{S}\right)\left\langle\phi_{d}\left|F_{\pi_{S}}\right| \phi_{d}\right\rangle,
\end{aligned}
$$

since only the same permutations are non zero as in Eq. (25). The two-electron operator $G$ is only a slightly more complicated. It is observed that the terms of $G$ are of two kinds, those for which $m$ and $n$ in $g_{m n}$ refer to coordinates within the same subset in $\phi_{d}$ and those for which $m$ and $n$ are in different subsets. We will symbolize the sum of all $g_{m n}$ totally within the $i$ th subset as $G(i)$ and we let $g\left(m_{i}, m_{j}\right) ; m_{i}=1,2, \cdots, l_{i}$; $m ;=1,2, \cdots, l ;$ be a particular term of the second kind. We then have

$$
\begin{aligned}
\left\langle\phi_{d}\left|G E^{(\mu)}(\gamma)\right| \phi_{d}\right\rangle=\frac{f_{(\mu)}}{|G|} \sum_{i=1}^{p} \sum_{\boldsymbol{x} S} P\left(\gamma, \pi_{S}\right)\left\langle\phi_{d}\left|G(i) \pi_{S}\right| \phi_{d}\right\rangle+ & \frac{f_{(\mu)}}{|G|} \sum_{i, j} \sum_{\boldsymbol{x}}\left\{P\left(\gamma, \pi_{S}\right)\left\langle\phi_{d}\left|g\left(m_{i}, m_{j}\right) \pi_{S}\right| \phi_{d}\right\rangle\right. \\
& \left.+P\left[\gamma,\left(m_{i} m_{j}\right) \pi_{S}\right]\left\langle\phi_{d}\left|g\left(m_{i}, m_{j}\right)\left(m_{i}, m_{j}\right) \pi_{S}\right| \phi_{d}\right\rangle\right\}
\end{aligned}
$$

21 The same can be said for the restricted Hartree-Fock treatment, but with even greater restrictions of the coefficients.

22 T. L. Gilbert, J. Chem. Phys. 43, S248 (1965).

${ }^{23}$ G. Das and A. C. Wahl, J. Chem. Phys. 44, 87 (1966). 
where $\left(m_{i}, m_{j}\right)$ is the permutation interchanging coordinates $m_{i}$ and $m_{j}$. We now have

$$
\frac{1}{2} l_{1} ! l_{2} ! \cdots l_{p} !\left(n^{2}+2-l_{1}^{2}-l_{2}^{2}-\cdots-l_{p}^{2}\right)
$$

different permutations yielding nonzero integrals in (28). This is less than $n !$ in most cases of interest and so the energy expression has been simplified as compared to the general case.

The one case mentioned above for which assumed orthogonality is not a restriction is for the operator $\theta N P N$. The orthogonality pattern of the pairing theorem ${ }^{24,25}$ may be assumed here with no effect on the energy. For all other $E^{(\mu)}(\gamma)$, assumed orthogonality will effect the calculated energy.

\section{APPENDIX A: EVALUATION OF $a(\pi)$}

In this appendix we derive a formula for $a(\pi)$ in the idempotent $\theta N P N$, based on the cycle structure of $\pi$. It is fairly evident that each cycle in $\pi$ makes its own contribution to the determination of $a(\pi)$ independent of the others, so we assume that $\pi$ contains at least one cycle of length $l$. We observe that $a(\pi)$ is present in the equation

$$
\theta N P N \pi N P N=a(\pi) N P N,
$$

and $a(\pi)=a\left(\nu^{-1} \pi \nu\right)$ where $\nu$ is any element of $N$. The cycle $\kappa=\left(i_{1}, i_{2}, \cdots, i_{l}\right)$ has one invariant under transformations by $\nu$. We have the equation

$$
\kappa=\left(i_{1} i_{2}\right)\left(i_{2} i_{3}\right) \cdots\left(i_{l-1} i_{l}\right),
$$

and it is observed that some of the interchanges may contain entries from different columns of $T$. We call such an interchange a crossing, and it is obvious the number of crossings is invariant to transformation by $\nu$. We denote this number by $n_{c}(\kappa)$. Some of the interchanges in (A2) may not be crossings so these are elements of $N$. These may be permuted all to the left (or right), and together form another element of $N$. There exists an element of $N, \nu_{0}$, which will bring $\kappa$ to the form

$$
\begin{aligned}
\nu_{0} \kappa \nu_{0}^{-1}=\nu[1,(n / 2)+S+ & 1][(n / 2)+S+1,2] \\
& \times[2,(n / 2)+S+2] \cdots,
\end{aligned}
$$

where number of interchanges after $\nu$ is $n_{c}(\kappa)$ and $\nu$ is an element of $N$. Because of the formula

$$
(i j)(j k)=(i k)(i j)
$$

Eq. (A3) becomes

$$
\begin{aligned}
\nu_{0} \kappa \nu_{0}^{-1}=\nu(1,2)(2,3) \cdots & {[1,(n / 2)+S+1] } \\
\times & {[2,(n / 2)+S+2] \cdots, }
\end{aligned}
$$

and $\kappa$ is equivalent to an element of $N$ times one of the

\footnotetext{
${ }^{24}$ A. T. Amos and G. G. Hall, Proc. Roy. Soc. (London) A263, 483 (1961).

${ }^{25}$ P.-O. Löwdin, J. Appl. Phys. Suppl. 33, 251 (1962).
}

Frg. 3. Tableau for the triplet case of 20 electrons.

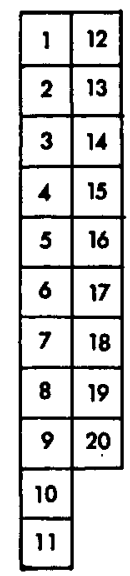

right coset generators. It is evident that the number of independent crossings in $\kappa$ is $\frac{1}{2} n_{c}(\kappa)$ or $\frac{1}{2}\left[n_{c}(\kappa) H\right]$ whichever is an integer, and this we denote by $\varphi_{c}(\kappa)$ and let $\varphi(\pi)$ be the sum of $\varphi_{\mathfrak{c}}$ for all cycles in $\pi$. If $\pi$ has the cycle structure $\left(1^{\alpha_{1}}, 2^{\alpha_{2}}, 3^{\alpha_{3}}, \cdots\right)$, then

$$
a(\pi)=(-1)^{n-\alpha_{1}-\alpha_{2}-\cdots \varphi(\pi)}\left(\begin{array}{c}
(n / 2)+S \\
\varphi(\pi)
\end{array}\right)^{-1} .
$$

As an example let us take the case where $n=20$, $S=1$,

$\pi=(1,12,2)(18,19,20)(3,4,16,17,11)$

$$
\times(5,13,6,14,7,15) \text {, }
$$

and one has the tableau in Fig. 3. We need merely count the number of times numbers for different columns are adjacent in each cycle. Thus

$$
\begin{aligned}
\varphi(\pi) & =\frac{2}{2}+\frac{0}{2}+\frac{2}{2}+\frac{5+1}{2}=5, \\
\alpha_{1}+\alpha_{2}+\cdots & =3+2+1+1=7, \\
a(\pi) & =+\left(\begin{array}{c}
11 \\
5
\end{array}\right)^{-1}=+\frac{1}{462} .
\end{aligned}
$$

\section{APPENDIX B: NUMBER OF INDEPENDENT FUNCTIONS FROM A FIXED BASIS}

We give here a derivation of the formula for the number of independent functions which can be constructed for $n$ electrons from a fixed basis of $m$ functions which was used in Sec. V.

If the multiplicity is $2 S+1$ then all product functions must contain at least $(n / 2)+S$ different spatial orbitals since otherwise the tableaux with repeated elements vanish. Let us choose $k$ of the $m$ functions with $(n / 2)+S \leq k \leq n$. There is a $k$-part partition of $n,\left(2^{n-k}, 1^{2 k-n}\right)$ which shows that $n-k$ functions must be repeated when placed in the tableau $\left(2^{(n / 2)-S}, 1^{2 S}\right)$. There are $\left(\begin{array}{c}n \\ n-k\end{array}\right)$ ways the pairing may be done for each of the $\left(\begin{array}{c}m \\ k\end{array}\right)$ ways the $k$ functions may be chosen 
from the $m$. The number of standard tableaux is just

$$
\frac{2 S+1}{2 k-n+1}\left(\begin{array}{c}
2 k-n+1 \\
k-S-(n / 2)
\end{array}\right)
$$

so we must multiply these three numbers together and sum over $k$ from $(n / 2)+S$ to $n$. Thus the total number of independent functions $N_{F}$ is

$$
\begin{aligned}
N_{F} & =\sum_{k=(n / 2)+S}^{n} \frac{2 S+1}{2 k-n+1}\left(\begin{array}{c}
m \\
k
\end{array}\right)\left(\begin{array}{c}
k \\
n-k
\end{array}\right)\left(\begin{array}{c}
2 k-n+1 \\
k-S-(n / 2)
\end{array}\right) \\
& =\frac{(2 S+1)}{(m+1)}\left(\begin{array}{c}
m+1 \\
(n / 2)+S+1
\end{array}\right)\left(\begin{array}{c}
m+1 \\
(n / 2)-S
\end{array}\right) .
\end{aligned}
$$

\section{APPENDIX C: IINEAR INDEPENDENCE OF $\theta$ NPN $\sigma_{1 k}$}

We here prove the theorem merely stated in Sec. IV of the article that the quantities

$$
\theta N P N \sigma_{1 k} ; \quad k=1,2, \cdots, f(\mu)
$$

are linearly independent in the group algebra. We here again define the $\sigma_{i k}$. Let $T_{1}, T_{2}, \cdots, T_{f}$ be standard tableaux for which the $k$ th one has the symbol $\alpha(k)_{i j}$ in the $i$ th row and $j$ th column. The tableaux $T_{k}$ are assumed ordered as follows:

$$
\alpha(k)_{i j}=\alpha\left(k^{\prime}\right)_{i j}
$$

for the rows $i=1,2, \cdots, i^{\prime}-1$, all columns, and for the $j=1,2, \cdots, j^{\prime}-1$ columns for the $i^{\prime}$ th row and then

$$
\alpha(k)_{i^{\prime} j^{\prime}}<\alpha\left(k^{\prime}\right)_{i^{\prime} j^{\prime}} \text { if } k<k^{\prime} .
$$

Also we let $P_{i}$ and $N_{i} ; i=1, \cdots, f$ be the positive and negative projectors corresponding to $T_{i}$. It is easily seen that if $\alpha\left(k^{\prime}\right)_{i^{\prime} j^{\prime}}>\alpha(k)_{i^{\prime} j^{\prime}}$ then $\alpha(k)_{i^{\prime \prime} j^{\prime \prime}}=\alpha\left(k^{\prime}\right)_{i^{\prime} j^{\prime}}$, where necessarily $i^{\prime \prime}>i^{\prime}$ and $j^{\prime \prime}<j^{\prime}$, i.e., this element appears in a later row and earlier column of $T_{k}$. It is also easily seen because of this that (see also Ref. 12)

$$
N_{i} P_{j}=P_{j} N_{i}=0 ; \quad j>i .
$$

Equation (C2) is the basis of our proof. Thus we examine

$$
\sum_{k=1}^{f} \gamma_{k} \theta N_{1} P_{1} N_{1} \sigma_{1 k}=0
$$

where $\sigma_{1 k}$ is the permutation $\sigma_{1 k}{ }^{-1} T_{1} \sigma_{1 k}=T_{k}$. Multiplying each term in (C3) on the right by $I$ in the form $\sigma_{1 k}\left(\sigma_{1 k}\right)^{-1}$ and on the left by $P_{l} N_{l}$ we get

$$
\begin{aligned}
0 & =\theta \sum_{k} \gamma_{k} \sigma_{1 k} N_{k} P_{k} N_{k} P_{l} N_{l}, \\
& =\theta^{\prime} \gamma_{l} \sigma_{1 l} N_{l} P_{l} N_{l}+\theta \sum_{k=l+1}^{f} \gamma_{k} \sigma_{1 k} N_{k} P_{k} N_{k} P_{l} N_{l}, \\
& =\theta^{\prime} \gamma_{f} \sigma_{1 f} N_{f} P_{f} N_{f} ; \quad l=f .
\end{aligned}
$$

As we let $l=f, f-1, f-2, \cdots, 1$ in turn we see that $\gamma_{f}, \gamma_{f-1}, \cdots, \gamma_{1}$ are each zero since $\sigma_{1 k} N_{k} P_{k} N_{k} \neq 0$. Thus the quantities are linearly independent.

THE JOURNAL OF CHEMICAL PHYSICS

VOL UME 50 , NUMBER 3

1 FEBRUAR Y 1969

\title{
Projected Hartree Product Wavefunctions. III. Comparison with the CI Method for $\mathrm{H}_{2}{ }^{*}$
}

\author{
R. C. Morrisoni and G. A. Gatluup \\ Department of Chemistry, University of Nebraska, Lincoln, Nebraska 68508
}

(Received 9 July 1968)

\begin{abstract}
Calculations of the energy of $\mathbf{H}_{2}$ have been performed using both the projected Hartree-Fock (PHF) method and a full CI method in the same basis for comparison of the two methods. It is discovered that PHF can account for $86 \%$ of the correlation energy, whereas CI will account for $88 \%$ of the correlation energy.
\end{abstract}

\section{INTRODUCTION}

In two recent articles ${ }^{1,2}$ Gallup has given a spinfree version ${ }^{3,4}$ of the projected Hartree-Fock (PHF)

\footnotetext{
* This article is based in part on a thesis submitted by R. C. M. to the Graduate Faculty in the University of Nebraska in partial fulfillment of the requirements for the Ph.D. degree.

† NASA Trainee 1966-1968.

1 G. A. Gallup, J. Chem. Phys. 48, 1752 (1968).

G. A. Gallup, J. Chem. Phys. 50, 1206 (1969), preceding article.

${ }^{3}$ F. A. Matsen, Advan. Quant. Chem. 1, 597 (1964).

4 R. D. Poshusta and R. W. Kramling, Phys. Rev. 167, 139 (1968).
}

method originally suggested by Löwdin. ${ }^{5}$ This is an extension of the independent-particle model in which optimization of the orbital functions in a different orbitals for different spins (DODS) trial function is done after the application of all projections necessary to insure the correct symmetry. Thus it has been emphasized by Goddard ${ }^{6}$ and in the first paper of this series, hereinafter denoted as $(\mathrm{I})^{1}$ that the use of single-particle orbitals with spatial dissymmetry and what might be called identity dissymmetry can

\footnotetext{
5 P.-O. Löwdin, Phys. Rev. 97,1509 (1955).

'W. A. Goddard, Phys. Rev. 157, 73, 81 (1967).
} 